Int. J. Morphol.,

36(3):1022-1027, 2018.

\title{
Uso de Terminologia Histologica en Textos de Histología Oral: Capítulo Esmalte
}

\author{
Use of Terminologia Histologica in Oral Histology Texts: Enamel Chapter
}

\author{
Javiera Navarrete ${ }^{1,2}$ \& Ignacio Roa Ra,3 $^{2,3}$
}

NAVARRETE, J. \& ROA, I. Uso de Terminologia Histologica en textos de histología oral: capítulo esmalte. Int. J. Morphol., 36(3):1022$1027,2018$.

RESUMEN: Para la enseñanza y aprendizaje de cualquier disciplina es clave el uso de una terminología clara y definida. Particularmente en el caso de Morfología, el rol de Programa Federativo Internacional de Terminología Anatómica (FIPAT) ha permitido avanzar a lograr la máxima unificación de la terminología asociada a esta disciplina, que considera múltiples variaciones lingüísticas en sus términos, dificultando su comprensión y difusión. Para que esta labor tenga un impacto más profundo en el uso correcto de la terminología internacional se debe articular un trabajo con los procesos de formación profesional que incorporan esta disciplina. El objetivo principal del presente estudio fue analizar la correcta utilización de Terminologia Histologica en los textos de estudios de Histología Oral, disponibles en Chile. Para este trabajo, se realizó un análisis de 4 textos de Histología Oral, dentro de los cuales se encontraban los 2 más utilizados por los estudiantes según las bases de datos de biblioteca de tres Universidades chilenas tanto públicas como privadas, además de 2 textos del área en idioma distinto al español (inglés y portugués). El análisis consistió, comparar el uso de los términos histológicos del capítulo "Esmalte" con los términos incluidos en Terminologia Histologica. Además de registrar errores en la denominación de términos tales como, eponimias, sinonimias y comparar con dos idiomas distintos al español, con el fin de descartar la traducción como problema principal. De los textos analizados el $100 \%$ presentaba una mayor cantidad de términos histológicos en comparación con los 14 descritos por Terminologia Histologica, de los cuales solo 7 son nombrados en la totalidad de los textos. Se encontraron 59 términos para las distintas estructuras del esmalte entre todos los textos, de éstos, solo 15 son nombrados conjuntamente en los cuatro textos y 7 poseen un término asignado en FIPAT. Además son descritas múltiples estructuras que no están construidas bajo las consideraciones de la FIPAT, presentando eponímias y sinonimias. Llama la atención que la mayoría de los términos extraídos no poseen representación en Terminologia Histologica además del uso predominante de epónimos y sinónimos tanto entre los textos de estudio como dentro de los mismos encontrándose veinte términos con más de un nombre. A partir de estos resultados se sugiere realizar las modificaciones pertinentes además de proponer focos para mejorar el uso de Terminologia Histologica en la formación de los futuros profesionales.

PALABRAS CLAVE: Terminologia Histologica; Histología oral; Esmalte; Epónimos.

\section{INTRODUCCIÓN}

Para la enseñanza y aprendizaje de cualquier disciplina es clave el uso de una terminología única, clara y definida (Hernández de la Rosa \& Gil Hernández, 2009). Particularmente en el caso de la Morfología, la terminología asociada ha evolucionado con el tiempo y los términos han sido objeto de considerable controversia y desacuerdo (Whitmore, 1999; Martin et al., 2009; Kachlik et al., 2015), es por ello que se ha intentado unificar a un lenguaje universal en múltiples ocasiones tanto en el área morfológica (Algieri et al., 2013; Roa \& del Sol, 2015; 2017; Fuentes \& Ottone, 2017), como en su aplicación quirúrgica (Duque \& Ríos, 2013; Duque et al., 2014).
Según Gutiérrez (2005), la utilización de un lenguaje correcto durante el proceso de aprendizaje de cualquier disciplina como por ejemplo la histológica, es realmente importante, debido principalmente a que resulta poco probable que un alumno pueda aprender una determinada disciplina o materia sin conocer el lenguaje en el que esa ciencia se expresa, contando además con la gran cantidad de palabras nuevas que deben aprender los alumnos de medicina en los tres primeros años de estudio. Asimismo, se considera que un mal aprendizaje de la terminología especializada constituye uno de los factores más importantes del fracaso académico de los alumnos.

\footnotetext{
${ }^{1}$ Escuela de Odontología, Universidad Autónoma de Chile, Temuco, Chile.

${ }^{2}$ Programa de Doctorado en Ciencias Morfológicas, Facultad de Medicina, Universidad de La Frontera, Temuco, Chile.

${ }^{3}$ Departamento de Ciencias Básicas Biomédicas, Facultad de Ciencias de la Salud, Universidad de Talca, Talca, Chile.

${ }^{4}$ Becario CONICYT-PCHA/Doctorado Nacional/2015-21150235.
} 
La utilización de términos incorrectos, sinonimias (Kachlik et al.) y epónimos en el vocabulario médico (Roa et al., 2016; Duque et al., 2017), y en textos utilizados por distintos estudiantes de carreras médicas, en conjunto con la discrepancia entre clínicos y morfólogos en la utilización del lenguaje, puede ser una fuente de confusión o un diagnóstico erróneo y tratamiento, que eventualmente puede ser pernicioso (Strzelec et al., 2017).

Este erróneo uso de la Terminología, ha sido reportado previamente en el desarrollo de textos de estudio de enseñanza secundaria, tal como es relatado por Caro et al. (2018), quienes reportan que el 11,43\% de la Terminología usada en el currículo de enseñanza de la biología en secundaria en Chile no se adhiere a Terminologia Anatomica Internacional promovida por el Programa Federativo Internacional de Terminología Anatómica (FIPAT). Resultados discordantes a los reportados por Park \& Park (2005), quienes luego de analizar 7 libros de texto de biología de educación secundaria y comprarlos con Terminologia Anatomica Internacional, determinaron que la concordancia de los términos de biología de la escuela secundaria con Terminologia Anatomica Internacional era 50,3 $\pm 2,7 \%$.

El rol del FIPAT ha permitido avanzar a lograr la máxima unificación de la terminología asociada a esta disciplina, que considera múltiples variaciones lingüísticas en sus términos, dificultando su comprensión y difusión. Para que esta labor tenga un impacto más profundo en el uso correcto de la terminología internacional se debe articular un trabajo con los procesos de formación profesional que incorporan esta disciplina (Losardo et al., 2015).

El objetivo del presente estudio fue analizar la correcta utilización y frecuencia de uso de Terminologia
Histologica en el capítulo "Esmalte" de textos de estudio de Histología Oral, disponibles en tres universidades de Chile.

\section{MATERIAL Y MÉTODO}

Fueron utilizados dos textos de Histología Oral, de las bibliotecas de tres Universidades chilenas; Universidad de Talca, Talca; Universidad de La Frontera, Temuco y Universidad Autónoma de Chile, Temuco. Los textos utilizados fueron: texto 1: "Histología, Embriología e Ingeniería Tisular Bucodental" (Gómez de Ferraris \& Campos Muñoz, 2009) y texto 2: "Principios de Histología y Embriología con Orientación Clínica" (Chiego Jr., 2014), los criterios de selección fueron: presencia de los textos en todas las instituciones, frecuencia de utilización por parte de los estudiantes y ediciones revisadas en español.

Realizamos un análisis de los términos histológicos citados en el capítulo "Esmalte". Para esto se identificó y registró el uso y frecuencia de todos los términos histológicos nombrados en este capítulo, incluyendo los encontrados tanto en el texto, como en figuras, cuadros y tablas. Los términos fueron comparados con aquellos incluidos «Terminologia Histologica: International terms for human cytology and histology», sección esmalte (FICAT, 2008). Además se registraron errores en la denominación de términos tales como, eponímias, sinonímias y ausencia de términos. Los resultados fueron contrastados con dos textos en idiomas distintos al español, texto 3: "Histologia e Embriologia Oral. Textos, Atlas, Correlações Clinicas”, en portugués (Katchburian \& Arana, 2012) y texto 4: "Ten Cate's Oral Histology. Development, Structure and Function" en inglés (Nanci, 2007) con el fin de descartar la traducción como problema principal.

Tabla I. Términos histológicos del capítulo Esmalte, encontrados en Terminologia Histologica: International terms for human cytology and histology, 2008.

\begin{tabular}{lll}
\hline & NOMINA LATINA & EQUIVALENTE ESPAÑOL \\
\hline H3.04.01.0.02011 & Enamelum & Esmalte \\
H3.04.01.0.02012 & Enameloblastus; Ameloblastus & Ameloblasto \\
H3.04.01.0.02013 & Prima enameli & Prisma del esmalte \\
H3.04.01.0.02014 & Membrana prismatica & Membrana prismática \\
H3.04.01.0.02015 & Stria transversa obscura & Estriación transversa oscura \\
H3.04.01.0.02016 & Crystallum hydroxyapatiti enameli & Cristales de hidroxiapatita del esmalte \\
H3.04.01.0.02017 & Processus ameloblasti & Proceso ameloblástico \\
H3.04.01.0.02018 & Cuticula dentis & Cutícula dental \\
H3.04.01.0.02019 & Lamella enamelea & Laminilla del esmalte \\
H3.04.01.0.02020 & Fusus enameli & Huso del esmalte \\
H3.04.01.0.02021 & Fasciculus enameli & Penacho del esmalte \\
H3.04.01.0.02022 & Junctio dentinoenameli & Unión amelodentinaria \\
H3.04.01.0.02023 & Linea incrementalis enamelea & Líneas incremental del esmalte \\
H3.04.01.0.02024 & Linea neonatalis & Línea neonatal \\
\hline
\end{tabular}




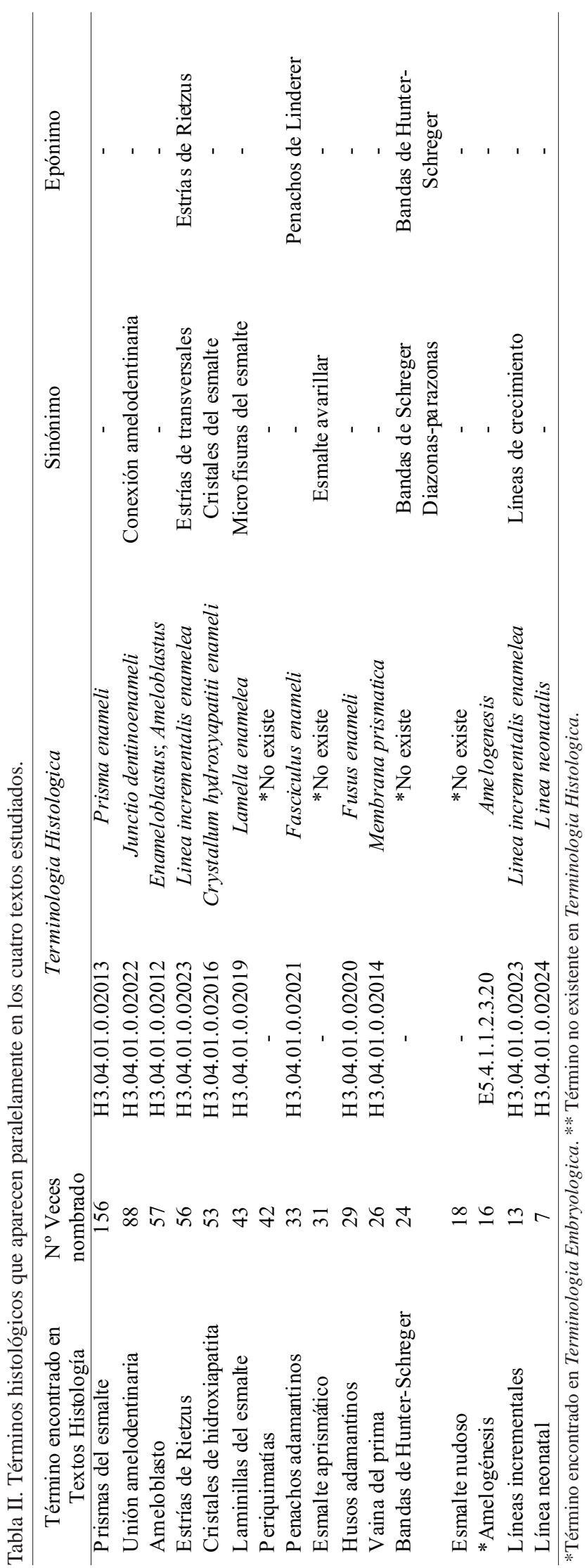

\section{RESULTADOS}

El análisis de los textos registró un total de 59 términos distintos para las diversas estructuras del esmalte. Siendo el texto 1 quien abarcó la mayoría de los registros con 57 términos, seguido por el texto 2 con 24 , así también se identificaron 22 términos para cada uno de los textos con idioma distinto al español, el texto 3 y el texto 4.

De la totalidad de los términos observados solo un $25,4 \%$ son nombrados paralelamente en los cuatro textos de estudio y solo un 13,9\% poseen un término asignado en la "Terminologia Histologica: International terms for human cytology and histology" (FICAT) (Tabla I). Es importante señalar que en esta última solo aparecen descritos 14 términos para las estructuras histológicas del esmalte, de éstos solo 8 aparecen mencionados en todos los textos, mientras que el resto es solo señalado en alguno de ellos (Tabla II).

Los 5 términos más mencionados entre los 4 textos son los prismas del esmalte, unión amelo-dentinaria, ameloblasto, estrías transversales oscuras y cristales de hidroxiapatita del esmalte, los cuales son mencionados en los textos con varios sinónimos y epónimos en algunos casos.

Respecto a las sinonimias el análisis reveló 20 términos con más de un nombre, habiendo estructuras que reciben hasta cuatro denominaciones dentro de un mismo texto como es el caso de las películas del esmalte en el texto 1 con nombres tales como: cutícula del esmalte, película secundaria, película adquirida y película exógena. Así también los prismas del esmalte que entre los distintos textos aparecen mencionados con tres nombres distintos (prismas del esmalte, varillas del esmalte y prismas adamantinos).

En cuanto al uso de epónimos se encontraron 8 estructuras histológicas que aún aparecen mencionadas en los textos con el nombre de aquellos investigadores que las describieron por primera vez; como por ejemplo las Estrías de Rietzus o los penachos de Linderer (Tabla III).

Con respecto al análisis de los textos 3 y 4 , estos presentaron errores similares a los encontrados en los textos 1 y 2 , existiendo sinonimias, eponimias y ausencia de términos, al ser comprados con Terminologia Histologica. 
Tabla III. Epónimos encontrados en los textos de Histología Oral.

\begin{tabular}{ll}
\hline \multicolumn{1}{c}{ Termino histológico } & \multicolumn{1}{c}{ Epónimo } \\
\hline Bandas claras y oscuras (Parazonas y diazonas) & Bandas de Hunter-Schreger \\
Penachos de adamantina & Penachos de Linderer \\
Relación esmalte-cemento & Casos de Choquet \\
Líneas de imbricación & Líneas de imbricación de Pickerill \\
Proceso ameloblástico & Proceso de Tomes \\
Cutícula/película primaria del esmalte & Membrana de Nasmyth \\
Líneas incremental del esmalte & Estrías de Rietzus \\
Línea neonatal & Línea de Rushton-Orban \\
\hline
\end{tabular}

\section{DISCUSIÓN}

El análisis realizado evidenció que la utilización de Terminologia Histologica en textos de Histología Oral es escaso. Llama la atención que la mayoría de los términos extraídos no poseen representación en Terminologia Histologica, también el uso predominante de epónimos y sinónimos tanto entre los textos de estudio como dentro de los mismos encontrándose 20 términos con más de un nombre, habiendo estructuras que reciben hasta cuatro denominaciones dentro de un mismo texto como es el caso de las película del esmalte (cutícula del esmalte, película secundaria, película adquirida, película exógena), así como, los prismas del esmalte que entre los textos aparecen mencionados de tres formas distintas (prismas del esmalte, varillas del esmalte, prismas adamantinos), erores coincidentes a los reportados por Vásquez \& del Sol (2015). Los 5 términos más mencionados entre los 4 textos son los prismas del esmalte, unión amelo-dentinaria, ameloblasto, estrías transversales oscuras y cristales de hidroxiapatita del esmalte, los cuales son mencionados en los textos con epónimos y varios sinónimos en algunos casos.

Tal problemática ha sido reportada por Caro et al. y Park \& Park quienes reportan falta de adhesión de los textos de secundaria en Chile y Corea respectivamente, con la Terminología Internacional, lo que indica que la problemática de un lenguaje morfológico no unificado se instaura en los estudiantes antes de que estos ingresen a pregrado.

Es realmente preocupante que ninguno de los cuatro textos se encuentre acorde con las directrices entregadas por Terminologia Histologica, a pesar de que tres (texto 1, 2 y 3 ) de los cuatro fueron publicados posterior al 2008, año de publicación de Terminologia Histologica: International terms for human cytology and histology (FICAT), la cual posteriormente (2011) fue publicada en línea por la Federación Internacional de Programas de Terminología Anatómica (FIPAT) perteneciente a la IFAA (Vásquez \& del Sol, 2014). Aunque antes del 2008 existía Nomina Histologica, el cual agrupaba entre otros los términos histológicos. Se hace necesario la publicación consensuada de Terminologías en español, con el fin de que estas sean utilizadas en ediciones posteriores.

Con respecto a la influencia de la traducción desde el latín, todos los textos analizados presentaron discrepancias con Terminologia Histologica, encontrándose epónimos (Estría de Retzius), sinonimias (Conexión amelodentinaria / Union amelodentinaria), ausencia de términos (Línea Neonatal), por lo cual descartamos una influencia real del idioma como principal fuente de los errores encontrados. Así como son múltiples los errores registrados en los textos de estudio, Terminologia Histologica presenta ausencia de algunos términos relevantes tales como periquematie (Roa \& del Sol, 2017), esmalte nudoso o Bandas claras y oscuras (de Hunter Schereger), las cuales juegan distintos roles dentro de la conformación histológica así como en la funcionalidad del esmalte. Otro término no encontrado en Terminologia Histologica en el capítulo Esmalte, es de amelogénesis, que se encuentra en Odontogénesis (E5.4.1.1.2.3.20 Amelogenesis), en Terminologia Embryologyca.

Las ciencias de la salud requieren de una comunicación clara y precisa, en cuanto a terminología (Vásquez \& del Sol, 2015), para todos los sectores profesionales y científicos implicados en la comunicación en el campo de la salud (Díaz Rojo, 2001). Vanos han sido los esfuerzos por divulgar y promover el uso de la Terminologia Anatomica, Histologica y Embryologica en el contexto clínico y educativo (Babinski et al., 2003; Silva Ortiz \& Torres Merchán, 2012), tal como lo hemos visto en nuestros resultados.

Según relata Montemayor Flores et al., (2016) se hace necesario establecer estrategias que favorezcan el uso de Terminologia tanto dentro como fuera de las aulas durante las lecciones de morfología y así abandonar el empleo de epónimos y sinónimos. Para tal fin es necesario el perfecto 
conocimiento de Terminologia, lo que facilita la comprensión y localización de las estructuras morfológicas, ya que el uso de los términos aceptados en ella permite al estudiante, al tiempo que aprende el uso del término, localizar la estructura morfológica nombrada y asociarla con la función, así el aprendizaje del extenso vocabulario morfológico puede ser realizado de una manera más comprensible, clara y simple, por este motivo, es importante que los profesores y las casas editoriales que publican no solo libros de las distintas áreas de la morfología sino de toda las ciencias de la salud, incorporen la Terminologia y abandonen el uso de epónimos, sinónimos y términos coloquiales, favoreciendo así el aprendizaje.

\section{CONCLUSIÓN}

La mayoría de los términos analizados no poseen representación en Terminologia Histologica. Existiendo uso predominante de epónimos y sinónimos tanto entre los textos de estudio como dentro de los mismos encontrándose 20 términos con más de un nombre; siendo estas denominaciones muchas veces poco descriptivas, pero si ampliamente recordadas por estudiantes y académicos. Es menester de los académicos resguardar el correcto uso de la Terminología, la cual describe a las estructuras morfológicas, proponiendo mejoras en los textos de estudio, así como de nuevos términos que estén más acorde con los señalado por la Federación Internacional de Asociaciones de Anatomía (IFAA) y la propia terminología.

NAVARRETE, J. \& ROA, I. Use of Terminologia Histologica in oral histology texts: enamel chapter. Int. J. Morphol., 36(3):10221027, 2018.

SUMMARY: For the teaching and learning of any discipline the use of a clear and defined terminology is key. Particularly in the case of Morphology, the role of the International Federative Program of Anatomical Terminology (FIPAT) has made it possible to advance towards the maximum unification of the terminology associated with this discipline, which considers multiple linguistic variations in its terms, hindering its understanding and dissemination. For this work to have a more profound impact on the correct use of international terminology, work must be articulated with the vocational training processes that incorporate this discipline. The main objective of the present study was to analyze the correct use of Terminologia Histologica in the texts of Oral Histology studies, available in Chile. For this work, an analysis of 4 texts of Oral Histology was carried out, among which were the 2 most used by students according to the library databases of three Chilean universities, both public and private, as well as 2 texts from the area. in a language other than Spanish (English and Portuguese). The analysis consisted in comparing the use of the histological terms of the "Enamel" chapter with the terms included in Terminologia Histologica. In addition to registering errors in the denomination of terms such as, eponymias, synonimies and compare with two languages ??other than Spanish, in order to rule out translation as the main problem. Of the texts analyzed, $100 \%$ had a greater number of histological terms compared to the 14 described by Terminologia Histologica, of which only 7 are named in all the texts. 59 terms were found for the different enamel structures among all the texts, of these, only 15 are named together in the four texts and 7 have a term assigned in FIPAT. In addition, multiple structures are described that are not constructed under the considerations of the FIPAT, presenting eponyms and synonyms. It is noteworthy that most of the extracted terms do not have representation in Terminologia Histologica besides the predominant use of eponyms and synonyms either, between the various texts or within them, and twenty terms can be found with more than one name. From these results it is suggested that pertinent modifications be made, in addition to proposing foci to improve the use of Terminologia Histologica in the training of future professionals.

KEY WORDS: Terminologia Histologica; Oral histology; Enamel; Eponyms.

\section{REFERENCIAS BIBLIOGRÁFICAS}

Algieri, R. D.; Ferrante, M. S. \& Pró, E. Analysis of the general surgeon's knowledge of International Anatomical Terminology (IAT). Int. J. Morphol., 31(4):1511-6, 2013.

Babinski, M. A.; Sgrott, E. A.; Luz, H. P.; Brasil, F. B.; Chagas, M. A. \& Abidu-Figueiredo, M. The relationship of the students with corpse in the pratical study of anatomy: The reaction and influence in the learning. Int. J. Morphol., 21(2):137-42, 2003.

Caro, G.; Roa, I.; Hormazabal Peralta, A. \& Lizana, P. A. Adherence to international anatomical terminology by a government-implemented high school biology science education curriculum. Int. J. Morphol., 2018. En Prensa.

Chiego Jr., D. J. Principios de Histología y Embriología Bucal. Con Orientación Clínica. $4^{\mathrm{a}}$ ed. Amsterdam, Elsevier, 2014.

Díaz Rojo, J. A. La terminología médica: Diversidad, norma y uso. Med. Interam., 20:34-8, 2001.

Duque, J. E. \& Ríos, J. Umbilical-spinous line: a morphological term that should be included in the anatomical terminology. Colomb. Med. (Cali), 44(2):202-4, 2013.

Duque, P. J. E.; Barco, R. J. \& Aguirre, C. V. E. De la terminología basada en epónimos en cirugía, a la Terminologia Anatomica Internacional: Un gran abismo que se puede evitar. Int. J. Med. Surg. Sci., 1(3):253-6, 2014.

Duque, P. J. E.; Barco, R. J. \& Vélez, G. J. F. Eponyms prevalence in publications and presentations at the 18th Congress of International Federation of Associations of Anatomists 2014. Int. J. Morphol., 35(2):525-8, 2017.

Federative International Comittee on Anatomical Terminology (FICAT). Terminologia Histologica, International Terms for Human Cytology and Histology. Philadelphia, Wolters Kluwer/Lippincott Williams \& Wilkins, 2008.

Fuentes, R. \& Ottone, N. E. Proposal on inclusion and elimination of anatomical terms in Terminologia Anatomica corresponding to the temporomandibular Joint. Int. J. Morphol., 35(1):12-5, 2017.

Gómez de Ferraris, M. E. \& Campos Muñoz, A. Histología, Embriología e Ingeniería Tisular Bucodental. $3^{\mathrm{a}}$ ed. Buenos Aires, Médica Panamericana, 2009. 
Gutiérrez, B. El Lenguaje de las Ciencias. Madrid, Gredos, 2005.

Hernández de la Rosa, Y. \& Gil Hernández, T. Importancia de la terminología dentro del proceso de comunicación científica. Edumecentro, 1(1):314. 2009.

Kachlik, D.; Musil, V. \& Baca, V. Terminologia Anatomica after 17 years: inconsistencies, mistakes and new proposals. Ann. Anat., 201:8-16, 2015.

Katchburian, E. \& Arana, V. Histología e Embriologia Oral. Textos, Atlas, Correlações Clinicas. $3^{\mathrm{a}}$ ed. Rio de Janeiro, Grupo Gen - Guanabara Kogan, 2012.

Losardo, R. J.; Prates, N. E. V. B.; Arteaga-Martínez, M.; Cabral, R. H. \& García-Peláez, M. I. International Morphological Terminology: More than anatomy, histology and embryology. Int. J. Morphol., 33(1):4007, 2015.

Martin, B. D.; Thorpe, D.; Barnes, R.; Deleon, M. \& Hill, D. Frequency in usage of FCAT-approved anatomical terms by North American anatomists. Anat. Sci. Educ., 2(3):94-106, 2009.

Montemayor Flores, B. G.; Herrera Vásquez, I. \& Soto Paulino, A. Analysis of the use of the Anatomical Terminology between students of anatomy courses at the Faculty of Medicine of the Universidad Nacional Autónoma de México. Int. J. Morphol., 34(4):1280-1284, 2016.

Nanci, A. Ten Cate's Oral Histology. Development, Structure and Function. $7^{\text {th }}$ ed. St. Louis, Elsevier Health Sciences, 2007.

Park, K. H. \& Park, J. Study on the differences between anatomical terminology and terminology of high school biology textbook. Kor. J. Anat., 38(4):307-14, 2005.

Roa, I. \& del Sol, M. Clarification of Terminologia Embryologica: Gartner duct. Int. J. Med. Surg. Sci., 2(2):481-6, 2015.

Roa, I. \& del Sol, M. Perikymata: A non-existent term. A scientific literature invention? Terminology analysis and proposal. Int. J. Morphol., 35(4):1230-2, 2017.

Roa, I.; Vásquez, B. \& Contreras, M. Eponyms persistence in Terminologia Histologica. Int. J. Morphol., 34(4):1245-52, 2016.

Silva Ortiz, S. R. \& Torres Merchán, N. Y. Significado del aprendizaje y la enseñanza de la anatomía: Contribuciones desde las percepciones de los estudiantes. Zon. Prox., (17):24-37, 2012.

Strzelec, B.; Chmielewski, P. P. \& Gworys, B. The Terminologia Anatomica matters: examples from didactic, scientific, and clinical practice. Folia Morphol. (Warsz), 76(3):340-7, 2017.

Vásquez, B. \& del Sol, M. Terminologia Anatomica and Terminologia Histologica. A meeting point between morphologists. Int. J. Morphol., 34(4):1585-90, 2015.

Vásquez, B. \& del Sol, M. The Terminologia Histologica in the Medical Sciences. Int. J. Morphol., 32(1):375-80, 2014.

Whitmore, I. Terminologia anatomica: new terminology for the new anatomist. Anat. Rec., 257(2):50-3, 1999.

\author{
Dirección de correspondencia: \\ Dr. Ignacio Roa Henríquez \\ Unidad de Morfología \\ Departamento de Ciencias Básicas Biomédicas \\ Facultad de Ciencias \\ de la Salud \\ Universidad de Talca Av. Lircay s/n \\ Talca \\ CHILE
}

E-mail: iroa@utalca.cl

Recibido: 25-04-2018

Aceptado:04-07-2018 